\title{
SUMMABILITY OF ALTERATIONS OF CONVERGENT SERIES
}

\author{
T.A. KEAGY \\ Mathematics Department \\ University of Texas at Tyler \\ Tyler, Texas 75701 U.S.A. \\ (Recelved February 29, 1980)
}

ABSTRACT. The effect of splitting, rearrangement, and grouping series alterations on the summability of a convergent series by $\ell-\ell$ and cs-cs matrix methods is studied. Conditions are determined that guarantee the existence of alterations that are transformed into divergent series and into series with preassigned sums. KEY WORDS AND PHRASES. $l-l$ method, es-cs method, rearrangement, splitting. 1980 MATHEMATICS SUBJECT CLASSIFICATION CODES. Primary -- 40C05, Secondary -- 40A25.

\section{INTRODUCTION.}

By an alteration of a series we will mean one of the three types: grouping, rearrangement, or splitting. The two types of summability methods we will be concerned with are cs-cs and $\ell-\ell$ methods. The sequence $x$ is in cs if $\left\{\sum_{i=1}^{n} x_{i}\right\}_{n=1}^{\infty}$ is convergent and in $\ell$ if $\sum_{i}\left|x_{i}\right|<\infty$. P. Vermes [1] characterized cs-cs methods A by the two properties 


$$
\sum_{p} a_{p q} \text { converges for each } q
$$

and

$$
\sup _{m} \sum_{q}\left|\sum_{p=1}^{m}\left(a_{p q}-a_{p, q-1}\right)\right|<\infty .
$$

Knopp and Lorentz [2] have characterized $\ell-\ell$ methods $A$ by the property

$$
\sup _{\mathrm{q}} \sum_{\mathrm{p}}\left|\mathrm{a}_{\mathrm{pq}}\right|<\infty \text {. }
$$

Each of the above methods is 1imit-preserving $\left(\sum_{q} x_{q}=\sum_{p}(A x)_{p}\right)$ whenever

$$
\sum_{\mathrm{p}}{ }_{\mathrm{pq}}=1 \text { for each } \mathrm{q} \text {. }
$$

The main purpose of this paper is to determine what effect alterations of a convergent series may have on the summability of the series under methods of the above type. In \$2, we describe each of the alteration types and investigate properties of the alterations and their respective matrix representations. In \$3, alterations of convergent serles that map to divergent series by cs-cs or $\ell-\ell$ transformations are determined. Finally, $\$ 4$ is concerned with alterations that are mapped by $\overline{c s}-c s$ or $\ell-\ell$ transformations to series that sum to a preassigned value $\sigma$.

\section{ALTERATIONS OF SERIES.}

By a grouping alteration of a series $\sum_{1} x_{1}$ we will mean a series $\sum_{1} y_{1}$ determined by an increasing sequence of positive integers $\{k(1)\}_{1=1}^{\infty}$ where the first term of the altered series is the sum of the first $k(1)$ terms of the original series, and for $1>1$

$$
y_{1}=\sum_{j=k(i-1)+1}^{k(i)} x_{j}
$$

Grouping alterations may be written in matrix form as transformations with all entries 0 or 1 that satisfy all requirements for limit-preserving cs-cs and $\ell-\ell$ methods. 
Rearrangements are perhaps the most famillar type of series alteration. They may be represented in matrix form as transformations with all entries 0 or 1 which have exactly one nonzero entry in each row and each column. Such maps are easily seen to be 1 imit-preserving $\ell-\ell$ transformations, but they need not necessarily be cs-cs. The answer to the question of precisely which rearrangements preserve the limit of all convergent series dates at least as far back as 1946 when Lev1 [3] first established that a rearrangement $p(1), p(2), p(3), \ldots$ of the positive integers will always yleld a convergent rearranged serles $\sum_{p}(k)=\sum a_{1}$ whenever $\left[a_{1}\right.$ is convergent if and only if there exists an integer $N$ such that for each $m$ the set of integers $p(1), \ldots, p(m)$ can be represented as the union of $N$ or fewer blocks of consecutive integers. Subsequent proofs of the same result were obtained by Agnew [4] and Guha [5]. Guha's proof is of particular interest here since he utilized (1.1) and (1.2) in his proof, a technique we will use in the proof of our Theorem 2 later.

Splittings of series were introduced by $P$. Wuyts [6]. If $\sum_{1} a_{1}$ is a series, then for each 1 we write $a_{1}=a(1,1)+\ldots+a\left(1, k_{1}\right)$. The resulting series

$$
a(1,1)+\ldots+a\left(1, k_{1}\right)+a(2,1)+\ldots+a\left(2, k_{2}\right)+\ldots
$$

is a splitting of the original series. In one way a splitting may be thought of as being the opposite of a grouping alteration since a grouping alteration produces a new series with sequence of partial sums a subsequence of the original series sequence of partial sums and a split series produces a supersequence of the original sequence of partial sums. The following theorem provides even more insight Into the connection between these two types of alterations.

THEOREM 1. Let $\sum_{1} x_{1}$ and $\sum_{1} y_{1}$ be two series. There exist a splitting $\sum_{1} a_{1}$ of $\sum_{1} x_{1}$ and a grouping alteration $\sum_{1} b_{1}$ of $\sum_{1} a_{1}$ such that $b_{1}=y_{1}$ for each 1 . PROOF. We first determine a splitting $\sum_{1} a_{1}$ of $\sum_{1} x_{1}$ by letting $a_{1}=y_{1}$, $a_{2 n}=x_{n}-a_{2 n-1}$, and $a_{2 n+1}=y_{n+1}-a_{2 n}$ for $n=1,2,3, \ldots$. Note that 
$a_{2 n-1}+a_{2 n}=x_{n}$ for each $n$. We now determine a grouping alteration $\sum_{1} b_{1}$ of $\sum_{1} a_{1}$ by letting $b_{1}=a_{1}$ and $b_{n+1}=a_{2 n}+a_{2 n+1}$. Then $b_{1}=a_{1}=y_{1}$ and $b_{n+1}=$ $\left(x_{n}-a_{2 n-1}\right)+\left(y_{n+1}-a_{2 n}\right)=y_{n+1}$ for $n>1$, hence the proof is complete. Representations of splittings in matrix format presents some difficulty if the series contains zero terms. If the series does not contain any zero terms, (2.1) may be expanded as

$$
a_{1}\left(b(1,1)+\ldots+b\left(1, k_{1}\right)\right)+a_{2}\left(b(2,1)+\ldots+b\left(2, k_{2}\right)\right)+\ldots
$$

where

$$
b(1,1)+\ldots+b\left(1, k_{1}\right)=1
$$

for each 1 . The splitting may now be represented in matrix form as a series to series transformation where each row has exactly one nonzero entry. The first $k_{1}$ entries of the first column will be $b(1,1), \ldots, b\left(1, k_{1}\right)$, and the $k_{1}+1$ through the $k_{1}+k_{2}$ entries of the second column will be $b(2,1), \ldots, b\left(2, k_{2}\right)$. The remaining colums are formed similarly.

THEOREM 2. The splitting matrix $B$ is cs-cs if and only if

$$
\sup _{n, k \leq k}|b(n, 1)+\ldots+b(n, k)|<\infty,
$$

and $B$ is $\ell-\ell$ if and only if

$$
\sup _{n}\left(|b(n, 1)|+\ldots+\left|b\left(n, k_{n}\right)\right|\right)<\infty .
$$

Furthermore, any splitting matrix that is cs-cs or $\ell-\ell$ is also limit-preserving.

PROOF. The proof follows from straightforward applications of (1.1), (1.2), (1.3), (1.4), and (2.2) together with the definition of a splitting matrix.

This yields a result reminiscent of, yet distinctly different from, the following theorem due to Wuyts [6].

THEOREM. (Wuyts) The split series (2.1) of a convergent series is itself convergent if and only if

$$
\lim _{n} \max _{k \leq k}|a(n, 1)+\ldots+a(n, k)|=0 .
$$




\section{MAPPINGS TO DIVERGENT SERIES.}

In [7] we provided an affirmative answer to the following question proposed by J.A. Fridy $[8]$ : is a null sequence necessarily in $\ell$ if there exists a sumpreserving $\ell-l$ matrix that maps all rearrangements of $x$ into $\ell$ ? similar question is as follows: If $x \in c s(l)$ and $A$ is a limit-preserving cs-cs $(l-l)$ matrix, does there exist an alteration of $x$ that $A$ fails to map into cs (l)?

Grouplng alterations are always 1imit-preserving cs-cs and $\ell-\ell$ transformations, therefore it is easy to see that if $A$ is a 1imit-preserving cs-cs ( $l-l)$ matrix and $x \in c s(l)$, then $A$ will map every grouping alteration of $x$ into cs (l)!

Rearrangements are also 1imit-preserving $\ell-\ell$ transformations, therefore it follows that $A$ will map every rearrangement of $x \in \ell$ into $\ell$ whenever $A$ is $\ell-\ell$. Rearrangements are not necessarily cs-cs as noted above. The following theorem resolves the question in case $x \in c s$ and $A$ is cs-cs.

THEOREM 3. Let $A$ be a 1imit-preserving cs-cs matrix and $x \in c s$ such that $\mathrm{x} \notin \ell$. There exists a rearrangement $\mathrm{y}$ of $\mathrm{x}$ such that $\mathrm{Ay} \notin \mathrm{cs}$.

PROOF. By (1.2) it is clear that each row of $A$ is of bounded variation and hence is convergent to some $L$. Suppose row $\mathrm{P}$ of $\mathrm{A}$ converges to $\mathrm{L} \neq 0$. We now construct a rearrangement $y$ of $x$ such that $\sum_{q} a_{p q} y_{q}$ fails to converge. Suppose the first $n$ terms of $y$ have been determined. Let $k=1+|L|\left(1+\max _{1}\left|x_{1}\right|\right)$ and $\mathrm{m}>\mathrm{n}+1$ such that

$$
\sum_{q=m}^{\infty}\left|a_{p q}-a_{p, q+1}\right|<|L| / 4 K
$$

and

$$
\left|\varepsilon_{p t}\right|=\left|a_{p t}-L\right|<|L| / 4 K
$$

for $t \geq m$. Choose $y_{1}=x_{j}$ where $j=\min \left\{q: x_{q}\right.$ is not one of $\left.y_{1}, \ldots, y_{1-1}\right\}$ for $\mathrm{n}<\mathrm{i}<\mathrm{m}$. Rearrange the terms of $\mathrm{x}$ not included in $\mathrm{y}_{1}, \ldots, \mathrm{y}_{\mathrm{m}-1}$ into a sequence $\left\{z_{i}\right\}_{i=m}^{\infty}$ such that 


$$
\sum_{1=m}^{\infty} z_{1}=2 /|L|+1
$$

and

Let $k>m$ such that $\left|\sum_{1-m}^{k}{ }_{1}\right|>2 /|L|$. Then

$$
\sup _{k}\left|\sum_{1-m}^{k} z_{1}\right|<2 R /|z| \text {. }
$$

$$
\begin{aligned}
\left|\sum_{q-m}^{k}{ }_{p q}^{z}\right| & \geq|L|\left|\sum_{q-m}^{k} z_{q}\right|- \\
& \sum_{q-m}^{k-1}\left|\varepsilon_{p q}-\varepsilon_{p, q+1}\right|\left|\sum_{1-m}^{q} z_{1}\right|- \\
& \left|\varepsilon_{p q}\right|\left|\sum_{1-m}^{k} z_{1}\right| \\
& >1 .
\end{aligned}
$$

Let $y_{1}=z_{1}$ for $m \leq 1 \leq k$ and continue this bullding process to obtain a rearrangement $y$ of $x$ such that for each $N$ there exist $n, m>N$ such that

$\left|\sum_{q=n}^{m} a_{p q}^{y} y_{q}\right|>1$.

Suppose now that each row of A 1s null. Let $\{y(1, j)\}_{j=1}^{\infty}$ be a rearrangement of $x$ such that $\sum_{q} y(1, q)=2$ and

$$
\sup _{n}\left|\sum_{q=1}^{n} y(1, q)\right|<M=3\left(\sup _{1}\left|x_{1}\right|+1\right) .
$$

Let $p(1)>0$ such that

$$
\left|\sum_{p=1}^{p(1)} \sum_{q}^{a} p q(1, q)-2\right|<1 / 4
$$

Choose $q(1)>0$ such that

$$
\left|\sum_{p=1}^{p(1)} \sum_{q=1}^{q(1)} a_{p q} y(1, q)-2\right|<1 / 4
$$

and

$$
\sup _{k \leq p}(1) 2 M \sum_{q=q(1)+1}^{\infty} \sum_{p=1}^{k}\left|\left(a_{p q}-a_{p, q+1}\right)\right|<1 / 8
$$

Let $y_{1}=y(1,1)$ for $1 \leq 1 \leq q(1)$. Let $y_{q(1)+1}=x_{f}$ where for $q=q(1)$

$$
j=\min \left\{1: x_{1} \text { is not one of } y_{1}, \ldots, y_{q}\right\} \text {. }
$$

Let $\{y(2, j)\}_{j=1}^{\infty}$ be a rearrangement of $x$ such that $y(2, q)=y_{q}$ for $1 \leq q \leq q(1)+1, \sum_{q} y(2, q)=0$, and $\sup _{n}\left|\sum_{q=1}^{n} y(2, q)\right|<M$. Let $p(2)>p(1)$ 
such that

$$
\left|\sum_{p=1}^{p(2)} \sum_{q} a_{p q}^{y(2, q)}\right|<1 / 8
$$

Choose $q(2)>q(1)+1$ such that

$$
\left|\sum_{p=1}^{p(2)} \sum_{q=1}^{q(2)} a_{p q} y(2, q)\right|<1 / 8
$$

and

$$
\sup _{k \leq p(2)} 2 M \sum_{q=q(2)+1}^{\infty} \sum_{p=1}^{k}\left|a_{p q}-a_{p, q+1}\right|<1 / 16 .
$$

Let $y_{1}=y(2,1)$ for $q(1)+1 \leq 1 \leq q(2)$ and $y_{q(2)+1}=x_{j}$ where $f$ is selected as in (3.3) for $q=q(2)$.

This selection process may be continued for $y$. Since $\sup _{n}\left|\sum_{q=1}^{n} y_{q}\right| \leq M$, it follows that $\sup _{n, m}\left|\sum_{q-n^{m}}^{m} y_{q}\right| \leq 2 M$. Therefore by the pattern established by (3.2) and (3.5), $\sum_{q} a_{p q} y_{q}$ will converge for each p. It also follows from (3.1) and (3.4) that the selection process for $y$ may be accomplished so that

$$
\left|\sum_{p=1}^{p(2 n)}(A y)_{p}-\sum_{p=1}^{p(2 n+1)}(A y)_{p}\right|>1
$$

for $n=0,1,2,3, \ldots$. Hence $A$ fails to map $y$ to $\mathrm{cs}$, and the proof is complete.

Splittings need not be cs-cs or $\ell-\ell$ maps. The following theorem leads to an answer to the question as to whether a limit-preserving cs-cs ( $l-l)$ matrix necessarily maps some splitting of every series $x$ in cs ( $($ ) into a series not in cs (l).

THEOREM 4. Let $A$.be a.matrix with an infinite number of nonzero columns, each one of which is in cs. If $x$ is a sequence, then there exists a splitting $y$ of $x$ such that Ay is not null.

PROOF. Suppose A has a row $k$ that is not eventually zero and without loss of generality assume no element of row $k$ is zero. Define a splitting $y$ of $x$ as follows: for $n=1,2,3, \ldots$ let $y_{3 n-2}=1 / a_{k, 3 n-2}, y_{3 n-1}=-y_{3 n-2}$, and $y_{3 n}=x_{n} \cdot$ Clearly $1 \lim _{n}\left|a_{k n} y_{n}\right| \neq 0$, and Ay fails to exist. 
Assume now that $A$ is row finite, and for row $p$ let $k(p)$ be the last nonzero element of row $p$. If row $p$ is a row of all zeros let $k(p)=0$. Let $p(1) \geq 1$ such that $k(p(1))>1$. Let $y_{1}=\ldots=y_{k(p(1))-1}=0, y_{k(p(1))}=1 / a_{p(1), k(p(1))}$, and $\mathrm{y}_{\mathrm{k}(\mathrm{p}(1))+1}=\mathrm{x}_{1}-\mathrm{y}_{\mathrm{k}(\mathrm{p}(1))}$.

Let $p(2)>p(1)$ such that $\left|\sum_{q=1}^{k(p(1))+1} a_{p(2), q} y_{q}\right|<1 / 2$ and $k(p(2))>$

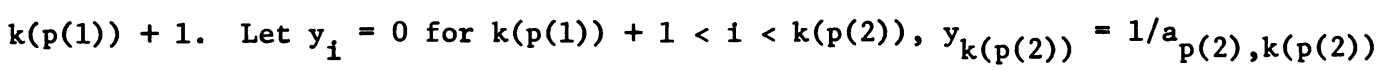
and $y_{k(p(2))+1}=x_{2}-y_{k(p(2))}$.

This selection process for $y$ may be continued so that $\mid$ (Ay) $_{p(i)} \mid>1 / 2$ for each 1 . It follows that Ay fails to be null, and the proof is complete.

COROLLARY 5. If $A$ is a limiti-preserving cs-cs $(l-\ell)$ matrix and $x \in c s(l)$, then there exists a splitting $y$ of $x$ such that Ay fails to be in cs $(l)$.

\section{MAPPINGS TO PREASSIGNED LIMITS.}

Rearrangement is the only series alteration method of our three types that can produce a new series with sum different from that of the original series. A more interesting question is the following: if $\mathrm{A}$ is a limit-preserving cs-cs $(\ell-\ell)$ matrix and $\sigma$ is a preassigned value, under what conditions can a convergent series be altered so that $A$ maps the altered series to one that sums to $\sigma$ ?

The answer for grouping alterations is easy since groupings are limitpreserving cs-cs and $\ell-\ell$ maps: the alteration exists only when $\sigma$ is the sum of the original series. The same answer applies when $A$ is $\ell-\ell, x \in \ell$, and the alteration is rearrangement. When $A$ is cs-cs, $x \in c s$ but $x \notin \ell$, and the alteration is a rearrangement, the desired alteration will always exist.

The answer for splittings is more complex and in fact depends on the particular matrix $A$ in question. If the limit-preserving cs-cs $(\ell-\ell)$ matrix is equivalent to convergence it is clear that A can map a splitting of the original series to a series with sum $\sigma$ only if $\sigma$ is the sum of the original series. 
THEOREM 6. Let $\mathrm{A}$ be a matrix with null rows and an increasing sequence of columns $\{q(i)\}_{i=1}^{\infty}$ such that

$$
\sum_{p} a_{p, q(i)}=1 \text { for } i=1,2,3, \ldots
$$

and

$$
\lim _{i}\left\{\sup _{n}\left|\sum_{p=1}^{n}\left(a_{p, q(2 i)}-a_{p, q(2 i-1)}\right)\right|\right\}=0 \text {. }
$$

If $x$ is any sequence with $\left\{\sum_{i=1}^{n} x_{i}\right\}_{n=1}^{\infty}$ bounded and $\sigma$ is any number, then there exists a splitting $\mathrm{y}$ of $\mathrm{x}$ such that Ay $\in \mathrm{cs}$ with sum $\sigma$.

PROOF. Let $\mathrm{y}_{\mathrm{q}(1)}=\sigma$ and $\mathrm{p}(1)>0$ such that if $\mathrm{t} \geq \mathrm{p}(1)$, then

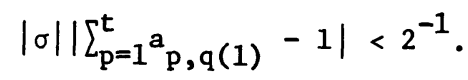

Using (4.2) and the fact that $A$ has null rows, choose $k(1)=q(2 i-1)$ such that $i>1$ and if $k(2)=q(2 i), y_{k(1)}=x_{1}-\sigma, y_{k(2)}=\sigma-x_{1}$, and $y_{i}=0$ otherwise for $i<k(2)$, then

$$
\sum_{i=1}^{2} \sum_{p=1}^{p(1)}\left|a_{p, k(i)} y_{k(i)}\right|<2^{-2}
$$

and

$$
\sup _{\mathrm{n}}\left|\sum_{\mathrm{p}=1}^{\mathrm{n}}\left(\mathrm{a}_{\mathrm{p}, \mathrm{k}(1)}-\mathrm{a}_{\mathrm{p}, \mathrm{k}(2)}\right)\right|<2^{-2} /\left(\left|\sigma-\mathrm{x}_{1}\right|+1\right) .
$$

Let $p(2)>p(1)$ such that if $t \geq p(2)$, then

$$
|\sigma|\left|\sum_{p=1}^{t} a, q(1)-1\right|<2^{-2}
$$

and

$$
\left|\sum_{\mathrm{p}=1}^{\mathrm{t}} \mathrm{a}_{\mathrm{p}, \mathrm{k}(1)} \mathrm{y}_{\mathrm{k}(1)}+\sum_{\mathrm{p}=1^{\mathrm{a}} \mathrm{a}, \mathrm{k}(2)}^{\mathrm{y}} \mathrm{y}_{\mathrm{k}(2)}\right|<2^{-3} \text {. }
$$

Again using (4.2) and the fact that $A$ has null rows, choose $k(3)=q(21-1)>$ $k$ (2) such that if $k(4)=q(2 i), y_{k(3)}=\sum_{i=1}^{2} x_{i}-\sigma, y_{k(4)}=\sigma-\sum_{i=1}^{2} x_{i}$, and $y_{i}=0$ otherwise for $k(2)<i<k(4)$, then

and

$$
\sum_{i=3}^{4} \sum_{p=1}^{p(2)}\left|a_{p, k(1)} y_{k(i)}\right|<2^{-3}
$$

$$
\sup _{n}\left|\sum_{p=1}^{n}\left(a_{p, k(3)}-a_{p, k(4)}\right)\right|<2^{-4} /\left(\left|\sigma-\sum_{i=1}^{2} x_{i}\right|+1\right) .
$$


This selection process for $y$ may be continued such that the pattern established by (4.3), (4.5), and (4.8) implies that for $n>p(1)$

$$
\left|\sum_{p=1}^{n} \sum_{q}^{a} q_{q}^{y} q_{q}-\sigma\right|<1,
$$

the pattern established by $(4.6),(4.7)$, and (4.9) Implies that for $n>p(2)$

$$
\left|\sum_{p=1}^{n} \sum_{q}^{a} p q_{q}^{y}-\sigma\right|<2^{-1}
$$

and in general for $n>p(k)$

$$
\left|\sum_{p=1}^{n} \sum_{q}^{a} q_{q}^{y_{q}}-\sigma\right|<2^{1-k} .
$$

COROLIARY 7. A sufficient condition for a limit-preserving cs-cs matrix to be stronger than convergence is that $A$ have null rows and satisfy (4.2).

COROLIARY 8. Let A be a limit-preserving c8-cs ( $l-l)$ matrix with null rows sat1sfying (4.2). If $x \in c 8(l)$ and $\sigma$ 1s any number, then there exists a splitting $y$ of $x$ such that Ay $\in c s$ with sum $\sigma$. If Instead of (4.2) A satisfies

$$
\lim _{i} \sum_{p}\left|a_{p, q(21)}-a_{p, q(21-1)}\right|=0,
$$

then a splitting $y$ of $x$ exists such that $A y \in l$ with sum $\sigma$.

COROLIARY 9. A sufficient condition for a limit-preserving $l$ - $l$ matrix to be stronger than convergence is that $A$ have null rows and satisfy (4.10).

\section{REFERENCES}

1. Vermes, P. Series to series transformations and analytic continuation by matrix methods, American J. Math. 71(1949) 541-562.

2. Knopp, R. and Lorentz, G. G. Beftrage zur absoluten Limitierung, Arch. Math. 2(1949) 10-16.

3. Lev1, F. W. Rearrangement of convergent series, Duke Math. J. 13(1946 579-585.

4. Agnew, R. P. Permutations preserving convergence of series, Proc. Amer. Math. Soc. 6(1955) 563-564.

5. Guha, U. C. On Levi's theorem on rearrangement of convergent series, Indian J. Math. 9(1967) 91-93.

6. Wuyts, P. On splitting the terms in a convergent series, Simon Stevin 11(1949) 180-188.

7. Keagy, T. A. Matrix transformations and absolute summability, Pacific J. Math. 63(1976) 411-415.

8. Fridy, J. A. Summability of rearrangements of sequences, Math. Z. $174(1975) \quad 187-192$. 


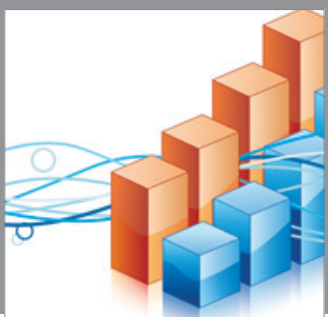

Advances in

Operations Research

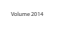

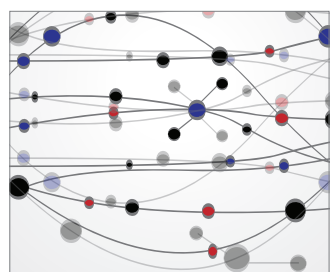

\section{The Scientific} World Journal
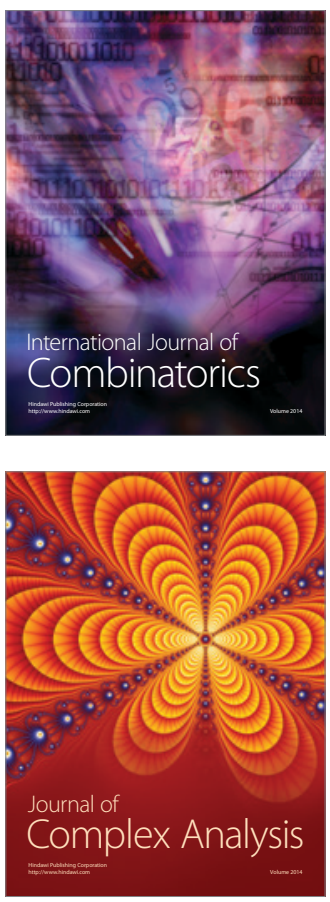

International Journal of

Mathematics and

Mathematical

Sciences
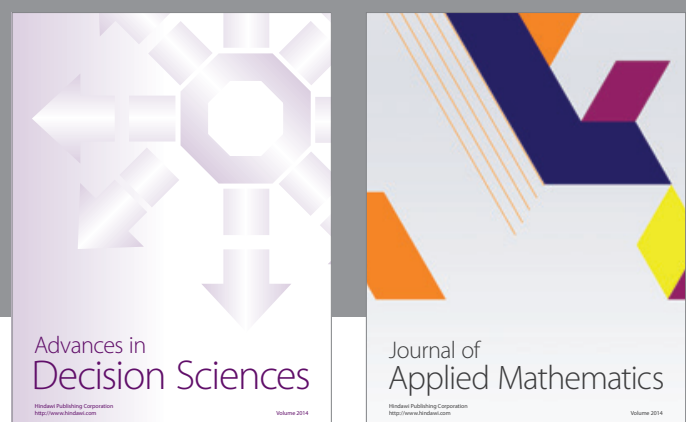

Journal of

Applied Mathematics
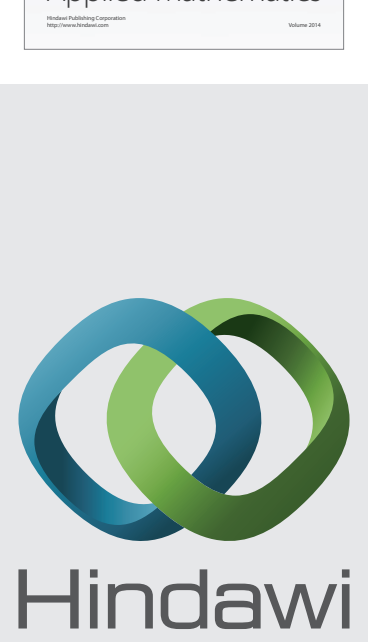

Submit your manuscripts at http://www.hindawi.com
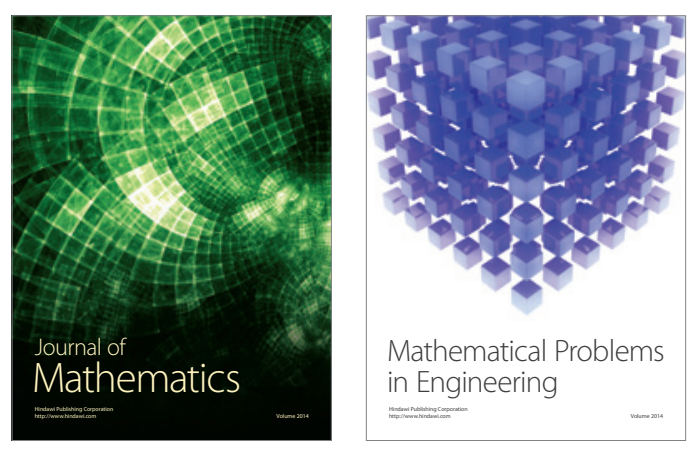

Mathematical Problems in Engineering
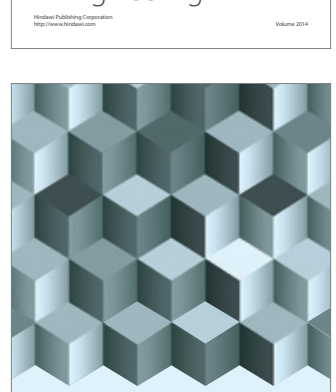

Journal of

Function Spaces
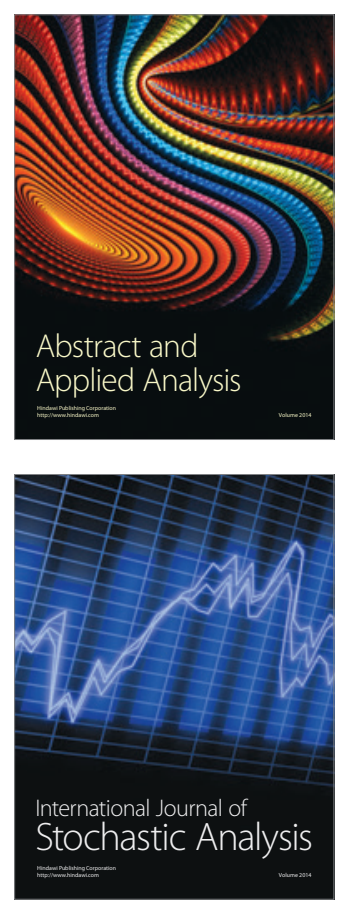

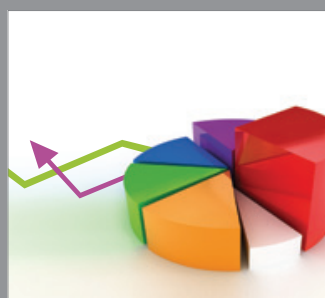

ournal of

Probability and Statistics

Promensencen
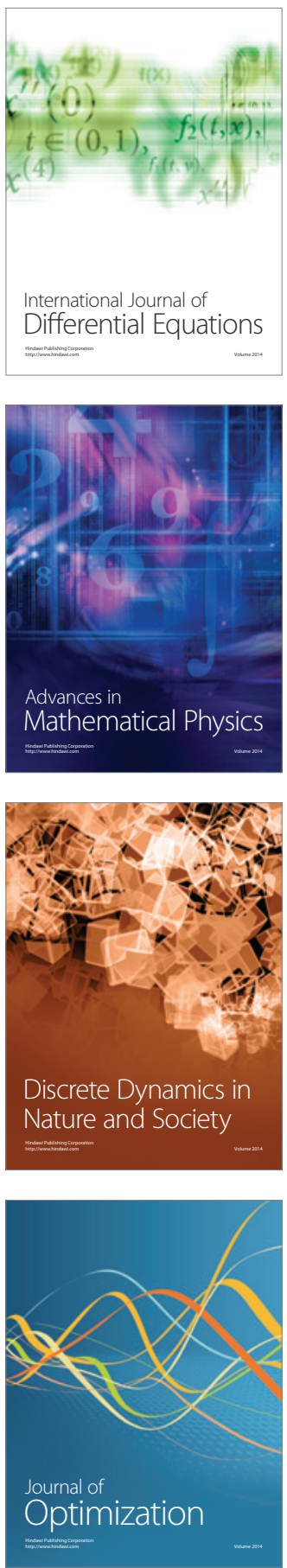